\title{
A Stochastic Programming Duality Approach to Inventory Centralization Games
}

\author{
Xin Chen* and Jiawei Zhang ${ }^{\dagger}$ \\ Submitted to Operations Research \\ First Version: February 24th, 2006
}

Revised: February 2nd, 2007; September 26th, 2007

\begin{abstract}
In this paper, we present a unified approach to study a class of cooperative games arising from inventory centralization. The optimization problems corresponding to the inventory games are formulated as stochastic programs. We observe that the strong duality of stochastic linear programming not only directly leads to a series of recent results concerning the non-emptiness of the cores of such games, but also suggests a way to find an element in the core. The proposed approach is also applied to inventory games with concave ordering cost. In particular, we show that the newsvendor game with concave ordering cost has a non-empty core. Finally, we prove that it is NP-hard to determine whether a given allocation is in the core for the inventory games even in a very simple setting.
\end{abstract}

Key Words: Stochastic Programming, Inventory Centralization, Cooperative Game.

\footnotetext{
${ }^{*}$ Department of Industrial and Enterprise Systems Engineering, University of Illinois at Urbana-Champaign. Email: xinchen@uiuc.edu. This work was supported by the National Science Foundation Grant CMMI-0653909.

${ }^{\dagger}$ Stern School of Business, IOMS-Operations Management, New York University, New York, NY, 10012. Email: jzhang@stern.nyu.edu. This work was partly supported by National Science Foundation Grant CMMI-0654116.
} 


\section{Introduction}

Consider a distribution system consisting of a set of retailers, which are facing random demands of a single product. The demands are generally correlated. It is well known in the inventory literature that inventory centralization leads to overall cost reduction or profit increase; see, for instance, Eppen [9]. In this setting, the retailers place orders before observing the demands, and after the demands are realized, excess inventory can be transhipped to retailers with excess demand.

There is a well developed literature on the analysis of inventory centralization. These analyses often assume a single decision maker who makes centralized decisions regarding inventory, allocation, and transhipment. However, many distribution systems consist of multiple independent decision makers who may collaborate with each other. For instance, Anupindi et al. [2] give several real life examples which involve alliances of independent retailers/distributors. One such example is the case of the machine tool builder Okuma America Corporation, which has 46 distributors in North and South America. Each of the distributors carries machine tools and selected repair parts in its inventory. When a customer orders an item that a distributor does not have, the distributor can identify the item's availability in the distribution network and arrange for intrachannel exchange of items electronically.

One important issue in such collaboration is to keep the different parties motivated to collaborate. The willingness to collaborate often depends on the existence of mechanisms that allocate the cost or gain (from the collaboration) in such a way that is considered advantageous by all the participants. (Even though collaboration leads to overall cost reduction, it is not always the case such mechanisms exist.) Indeed, getting all parties to agree on how to share costs and benefits was identified as one of the major barriers to collaborative commerce in practice (see $[8,26]$ ).

In this paper, we analyze the cost allocation issue in the inventory centralization setting using the notion of core, a basic solution concept in cooperative game theory. Roughly speaking, a core is a set of cost allocations under which no group of retailers should be charged more than they would pay if they were to separate and follow an optimal strategy for themselves. That is, no group of retailers will be better off by deviating from the cooperation. Thus, allocating cost according to a core allocation may create incentives for retailers to cooperate. However, in general, determining the non-emptiness of a core is not straightforward. Even in cases where a core can be shown to be 
nonempty, identifying an allocation in the core is usually challenging.

We refer to our cost allocation problem as a (cooperative) inventory centralization game. In this paper, we mainly focus on the existence of core allocations of inventory centralization games. In case a core allocation exists, we also discuss the question of how to find one.

\subsection{Closely related research}

Some special cases of inventory centralization games have been studied in the literature. One special case is called the newsvendor game, in which each retailer is a newsvendor with identical cost structures and the transportation cost associated with re-allocating inventory after observing the demand is ignored (see Gerchak and Gupta [10]). Hartman et al. [17] show that newsvendor games have non-empty cores under special assumptions on demand distributions. This result has been generalized independently by Müller et al. [23] and Slikker et al. [35], who show that the core is always non-empty regardless of the demand distributions. The result is still valid even when there are infinitely many retailers, as proved by Montrucchio and Scarsini [22]. Moreover, newsvendor games are not concave in general (for the definition of a concave game, please refer to Subsection 2.1). A very recent paper by Ozen et al. [29] discusses the convexity of the newsvendor games under special assumptions about the demand distributions.

More general inventory centralization games have been studied by Slikker et al. [36] and by Ozen et al. [28], who extend the newsvendor game in two ways. First, they consider transportation costs in their models. Second, in their settings, the cost parameters of the retailers can be different. Although the models are much more complicated than the one studied in Hartman et al. [17], Slikker et al. [36] and Ozen et al. [28] still manage to show that the games have non-empty cores.

Anupindi et al. [2] study a two-stage model that is closely related to the one in Slikker et al. [36]. In their model, the retailers do not fully cooperate. In the first stage, before demand realization, each retailer makes its own decision on how much to order. In the second stage, after observing the demands, the retailers can cooperate by reallocating their inventories. Granot and Sosic [13] analyze a similar problem but allow retailers to decide how much inventory to share with others.

The notion of core has been used to analyze cost allocation problem in other inventory man- 
agement models as well, including lot-sizing models (van den Heuvel et al. [40], Chen and Zhang [4]) and joint replenishment models (Meca et al. [21], Anily and Haviv [1], Dror and Hartman [6], and Zhang [41]).

Applications of game theory in analyzing cooperations among supply chain agents can also be found in Nagarajan and Sošić [24]. For a comprehensive review, see Nagarajan and Sošić [25].

\subsection{Our results and approach}

The goal of our paper is to present a unified approach to analyze the inventory centralization games considered in $[17,23,35,36,28]$ using the duality theory of stochastic programming developed by Rockafellar and Wets [33]. To illustrate the basic idea, we will mainly focus on the game proposed by [28]. However, it is important to point out that the stochastic programming approach can be applied in settings well beyond [28].

Our main results can be summarized as follows.

- We formulate the inventory centralization problem as a stochastic linear program. Then we show that, the existence of the core of the general inventory game studied in [28] follows directly from the strong duality of stochastic linear programming. This results in a much simpler proof than that in [28]. Moreover, the stochastic linear programming approach to the inventory games offers a constructive proof to the non-emptiness of the core, i.e., a core allocation can be defined by any given optimal dual solution. For a special case, i.e., the newsvendor game studied in [17] and [23], the dual admits a simple closed-form solution.

- We further illustrate our duality approach in a setting where the cost structure is non-convex and thus the strong duality theory of Rockafellar and Wets [33] may not directly apply. In particular, we consider inventory games with concave ordering cost, which reflects possible quantity discounts. We show that if the dual solution has certain property, then the game has an empty core. Furthermore, we prove this property always holds for inventory games with concave ordering cost, and thus it has a non-empty core. We remark that, although the dual is in the form of infinite dimensional stochastic linear program, it admits a closed-form solution. Constructing such a dual solution only requires finding an optimal ordering quantity for the newsvendor problem with concave ordering cost. 
- Finally, we show that even in a very simple setting, determining whether an allocation is in the core of the newsvendor game is NP-hard. Therefore, this NP-hardness result applies to other inventory centralization games as well. We include this result mainly for the completeness of the paper, because testing membership of the core for a given allocation has been a standard research question in (computational) cooperative game theory; see, for instance, Deng and Papadimitriou [5] and Faigle et al. [7].

Now we briefly discuss our results and approach. Our approach is motivated by the work of Owen [27], who used linear programming duality to show the non-emptiness of the core for the (deterministic) linear production game. Owen's approach has became one of the systematic tools in analyzing cooperative games and has found numerous applications; see, for instance, Granot [12] and Tamir [38]. The allocation defined by a dual solution is often called Owen's point, which has became subjects of many papers in cooperative game theory. Therefore, although we focus only on inventory centralization games in this paper, we expect that the stochastic programming duality approach will find more applications in analyzing cooperative games with uncertainty.

The duality approach has other advantages as well. For instance, provides a constructive proof for the non-emptiness of the core for inventory centralization games. This is in contrast with the existence proofs of previous works in this area. Since the first submission of our paper, we became aware of the paper by Montrucchio and Scarsini [22], which proposes an explicit core allocation for the newsvendor game when the demand distribution is continuous. Recall that the newsvendor game assumes identical cost structures and no transportation cost. It is interesting to notice that, for this special case, the core allocation suggested in [22] is captured by the allocation defined by the dual solution. Moreover, it is not clear how the constructive proof in [22] can be generalized for other inventory centralization games such as those in $[36,28]$. On the other hand, our approach is applicable to much more general settings; please see the discussion in Section 6 .

From a computational point of view, one needs to solve the dual of a two-stage stochastic linear program in order to compute a core allocation. This might be considered as one drawback of the stochastic programming duality approach, especially when the size of the stochastic linear program is too large. Nonetheless, the duality approach provides a way to compute a core allocation, at least for problems of moderate sizes. Future development of stochastic programming and computing technologies might make it possible to solve large scale stochastic linear programs more efficiently. 
Further, high quality near-optimal solution to two-stage stochastic linear programs can be obtained by using sampling techniques $[34,20]$. Therefore, an approximate core allocation may be computed relatively efficiently. This could be sufficient for many applications. Since the purpose of this paper is mainly to suggest a systematic approach to analyze cooperative games using stochastic programming duality, we do not get into details of solving stochastic linear program.

The rest of the paper is organized as follows. In Section 2, we introduce the model, the associated inventory game, and the solution concepts in cooperative game theory. In Section 3, we use the duality of stochastic linear programming to derive the result for inventory games with linear ordering cost. In section 4, we develop a strong duality result for the newsvendor problem with concave ordering cost, and employ it to show that the core of the corresponding game is non-empty. In Section 5, we prove the NP-hardness of testing the membership in the core of the newsvendor game. Finally, we discuss possible applications of the duality approach in Section 6 .

\section{Preliminaries}

\subsection{Cooperative Games}

Here we briefly introduce the basic concepts of cooperative game theory that will be used in this paper. Let $N=\{1,2 \cdots, n\}$ be the set of players. A collection of players $S \subseteq N$ is called a coalition. The set $N$ is sometimes referred to as the grand coalition. A characteristic cost function $C(S)$ is defined for each coalition $S \subseteq N$, which could be the minimum total cost that coalition $S$ should pay if the members of $S$ decide to secede from the grand coalition and cooperate only among themselves. A cooperative game is determined by the pair $(N, C)$. For each subset $S \subset N$, the cooperative game $(S, C)$ is called a subgame. The game $(N, C)$ is called a concave game if for every pair of subsets $S, T \subseteq N, C(S)+C(T) \geq C(S \cup T)+C(S \cap T)$.

Given a cooperative game, there are many ways to divide the cost (or the value) of the game among the players. The cost allocation issue has been extensively studied in the literature of cooperative game theory. In this paper, we focus on the so-called core allocations, which will be defined below.

A vector $l=\left(l_{1}, l_{2}, \cdots, l_{N}\right)$ is called an imputation of the game $(N, C)$ if $\sum_{j \in N} l_{j}=C(N)$ and 
$l_{j} \leq C(\{j\})$ for every $j \in N$. One can interpret an imputation as a division of $C(N)$ that charges every player at most as much as she will pay by herself. When we generalize this idea to every coalition of players, we get the notion of core.

Definition 1. An allocation $l$ is in the core of the game $(N, C)$, if $\sum_{j \in N} l_{j}=C(N)$ and for any subset $S \subseteq N, \sum_{j \in S} l_{j} \leq C(S)$.

Roughly speaking, a core is a set of cost allocations under which no coalition should be charged more than they would pay if they were to separate and follow an optimal strategy for themselves. That is, no coalition will be better off by deviating from the grand coalition. If the core of a game is non-empty, then there is at least one allocation of the cost that is considered advantageous by all the players.

It is well-known that the core of a concave game is always non-empty. It is also known that the newsvendor game is not concave in general [29].

\subsection{The Inventory Model}

We consider a distribution system consisting of a supplier, a set of $m$ warehouses denoted by $W=\{1,2, \cdots, m\}$, and a set of $n$ retailers denoted by $N=\{1,2, \cdots, n\}$. The retailers sell a single type of goods. Each retailer $j \in N$ faces a random demand $d_{j}(\omega)$ with $\mathrm{E}\left[d_{j}(\omega)\right]<\infty$ where uncertainty is represented in terms of random experiments with outcomes denoted by $\omega$. The set of all outcomes is denoted by $\Omega$. Also, each retailer $i$ operates a set of warehouses $Z_{j} \subseteq W$. If a subset $S \subseteq N$ of retailers forms a coalition, then any retailer in $S$ is allowed to use any warehouse in $\cup_{j \in S} Z_{j}$, i.e., the set of warehouses that are operated by the retailers in $S$. Finally, for any coalition $S$, let $d^{S}(\omega)=\left(d_{j}(\omega)\right)_{j \in S}$ and $d^{S}=\left(d_{j}\right)_{j \in S}$, where $d_{j}$ is the realized demand of retailer $j$.

The sequence of events happens as follows. At the beginning, before observing the random demands, each warehouse $i$ associated with retailers in the coalition $S$ orders certain amount of goods, say $y_{i}$, from the supplier by paying a cost of $c_{i}$ per unit. Since we are considering a single period problem, the lead time can be assumed without loss of generality to be zero.

Then, the demands are realized and the realized total demand of coalition $S$ is denoted as $d=\left(d_{1}, d_{2}, \cdots, d_{n}\right)^{T}$. After the realization of demands, all goods at the warehouses are allocated 
to the retailers, say $x_{i j}$ units of goods are shipped from warehouse $i$ to retailer $j$. The transportation cost of sending one unit good from $i$ to $j$ is $s_{i j}$. For each retailer $j$, if the total amount of goods received from the warehouses is more than the realized demand $d_{j}$, it pays a per unit holding cost of $h_{j}$ for excess inventory; on the other hand, the unsatisfied demands are lost and retailer $j$ pays a per unit penalty cost of $p_{j}$ for lost sales.

We remark that each coalition is responsible for not only the transportation cost, inventory holding cost, and penalty cost incurred at the retailers in the coalition, but also the ordering cost incurred at the associated warehouses. The coalition makes the decision on the order quantity at each warehouse.

In this model, $y_{i}, i=1,2, \cdots, m$ is the first stage decision variable. After the demands are realized, a recourse decision should be made, which is the amount of goods sent from warehouse $i$ to retailer $j$, i.e., $x_{i j}$. For the coalition $S \subset N$, the goal is to minimize the expected total cost for the coalition, which includes the ordering cost, transportation cost, inventory holding cost, and penalty cost.

Denote the minimum expected cost of the coalition $S$ by $C(S)$, which is the optimal value of the following two-stage stochastic linear program.

$$
\begin{aligned}
C(S)= & \min \sum_{i \in \cup_{j \in S} Z_{j}} c_{i} y_{i}+\mathrm{E}\left[f\left(y, d^{S}(\omega)\right)\right] \\
\text { s.t. } & y_{i} \geq 0, i \in \cup_{j \in S} Z_{j}
\end{aligned}
$$

where $y=\left(y_{i}\right)_{i \in \cup_{j \in S} Z_{j}}$, and $f\left(y, d^{S}\right)$ is defined by

$$
\begin{aligned}
f\left(y, d^{S}\right):=\min & \sum_{j \in S} p_{j} z_{j}+\sum_{j \in S} h_{j} I_{j}+\sum_{i \in \cup_{j \in S} Z_{j}, j \in S} s_{i j} x_{i j} \\
\text { s.t. } \quad & z_{j}+\sum_{i \in \cup_{j \in S} Z_{j}} x_{i j} \geq d_{j}, \quad j \in S \\
& I_{j}-\sum_{i \in \cup_{j \in S} Z_{j}} x_{i j} \geq-d_{j}, \quad j \in S \\
& y_{i}-\sum_{j \in S} x_{i j}=0, \quad i \in \cup_{j \in S} Z_{j} \\
& z_{j}, I_{j}, x_{i j} \geq 0,
\end{aligned}
$$

where $z_{j}$ and $I_{j}$ denote the lost sales and excess inventory, respectively. In the second stage formulation, the first constraint implies that unsatisfied demand is lost, the second constraint 
corresponds to the excess inventory, and the third constraint implies that the warehouses will not hold any inventory at the end.

The cooperative inventory game is the pair $(N, C)$ where the characteristic function $C$ is defined by (1).

Remark 1. As mentioned in [28], the model described above is general enough to capture the case where there is no warehouse and only retailers can place the orders. To see this, we let $W=N$, and for each $j \in N$ we let $Z_{j}=\{j\}$. Therefore, if only a subset of retailers $S$ cooperates, they can re-allocate the goods among themselves.

Remark 2. The assumption that the warehouses do not hold inventory is not critical and merely for the ease of presentation. In fact, for each warehouse $i$, we can consider an auxiliary retailer $j(i)$ attached to it. The demand of retailer $j(i)$ and the transportation cost $s_{i, j(i)}$ are both set to be zero. Further, the holding cost at retailer $j(i)$ is the same as that at warehouse $i$. In this new distribution system, any inventory held at warehouse $i$ can be assumed to be held at retailer $j(i)$. Therefore, we can assume without loss of generality that the warehouses do not hold any inventory.

Remark 3. In this inventory game, we focus only on the allocation of the expected cost. One may wonder whether it is possible to derive a stable allocation of the actual cost for each demand realization. More precisely, let's assume that $x(S)$ is an optimal decision (before the realization of the random demand) to the coalition $S$. Assume that the cost of coalition $S$, under optimal decision $x(S)$, is $C(S, x(S), \omega)$ in scenario $\omega$. Therefore, it would be nice if we can find an allocation $\left(l_{j}(\omega): j \in N\right)$ for each scenario $\omega$ such that

$$
\sum_{j \in N} l_{j}(\omega)=C(N, x(N), \omega), \quad \sum_{j \in S} l_{j}(\omega) \leq C(S, x(S), \omega) \quad \forall S \subseteq N
$$

If such an allocation exists, it implies that we can allocate the actual cost for each demand realization, and the allocation is stable in every scenario. Unfortunately, in [16], Hartman and Dror has shown that such an allocation does not exist in general, even for the newsvendor game. On the other hand, it is easy to see that, if there is a core allocation $\left(l_{j}: j \in N\right)$ for the (expected cost) game $(N, C)$, then we can define

$$
l_{j}(\omega)=\tau_{j} C(N, x(N), \omega)
$$


where

$$
\tau_{j}=\frac{l_{j}}{\sum_{k \in N} l_{k}}
$$

It is straightforward to verify that

$$
\sum_{j \in N} l_{j}(\omega)=C(N, x(N), \omega), \quad \sum_{j \in S} \mathrm{E}\left[l_{j}(\omega)\right] \leq \mathrm{E}[C(S, x(S), \omega)] \quad \forall S \subseteq N .
$$

That is, before the demand is realized, the retailers can agree on a cost allocation rule, which allocates the actual cost in each scenario, and the allocation is stable with respect to the expected cost of each coalition.

\section{Inventory Games with Linear Ordering Cost}

\subsection{Dual of the inventory problem}

Problem (1) is a special case of the following two-stage stochastic linear program

$$
\begin{array}{ll}
\min & \nu_{1}^{T} x_{1}+\mathrm{E}\left[g\left(x_{1}, \omega\right)\right] \\
\text { s.t. } & A_{11} x_{1}=b_{1} \\
& x_{1} \geq 0
\end{array}
$$

where

$$
\begin{array}{ll}
g\left(x_{1}, \omega\right)=\min & \nu_{2}^{T} x_{2} \\
\text { s.t. } & A_{12} x_{1}+A_{22} x_{2}=b_{2}(\omega) \\
& x_{2} \geq 0
\end{array}
$$

The dual of problem (2) is

$$
\begin{array}{ll}
\max & \mathrm{E}\left[b_{1}^{T} \pi_{1}+b_{2}^{T}(\omega) \pi_{2}(\omega)\right] \\
\text { s.t. } & A_{11}^{T} \pi_{1}+\mathrm{E}\left[A_{12}^{T} \pi_{2}(\omega)\right] \leq \nu_{1} \\
& A_{22}^{T} \pi_{2}(\omega) \leq \nu_{2} .
\end{array}
$$

If the set $\Omega$ is finite, problem (2) is just a linear program, and the strong duality of linear programming applies if the problem is feasible. However, for general $\Omega$, the situation is more complicated. Rockafellar and Wets [33] presented conditions under which the optimal values of the primal and the dual are equal. In order to introduce their result, we need the following definition. 
Definition 2. The stochastic program (2) is said to have relatively complete recourse if for any $x_{1} \in\left\{x_{1}: A_{11} x_{1}=b_{1}, x_{1} \geq 0\right\}, \mathrm{E}\left[g\left(x_{1}, \omega\right)\right]<\infty$.

Then the results of Rockafellar and Wets [33] imply the following theorem (see also Korf [19], Theorem 1.3).

Theorem 1. If (2) is strictly feasible and has relatively complete recourse, then the optimal value of (2) is equal to the optimal value of (3).

Now we apply Theorem 1 to the inventory centralization problem (1) under investigation. It is easy to see that the dual problem of (1) is

$$
\begin{array}{ll}
\max & \mathrm{E}\left[\sum_{j \in S}\left(\alpha_{j}(\omega)-\beta_{j}(\omega)\right) d_{j}(\omega)\right] \\
\text { s.t. } & \mathrm{E}\left[\gamma_{i}(\omega)\right] \leq c_{i}, \quad i \in \cup_{j \in S} Z_{j} \\
& \alpha_{j}(\omega) \leq p_{j}, \quad j \in S, \omega \in \Omega \\
& \beta_{j}(\omega) \leq h_{j}, \quad j \in S, \omega \in \Omega \\
& \alpha_{j}(\omega)-\beta_{j}(\omega)-\gamma_{i}(\omega) \leq s_{i j}, \quad j \in S, i \in \cup_{j \in S} Z_{j}, \omega \in \Omega \\
& \alpha_{j}(\omega) \geq 0, \beta_{j}(\omega) \geq 0, \quad \omega \in \Omega,
\end{array}
$$

which can be further simplified as

$$
\begin{aligned}
\max \quad & \mathrm{E}\left[\sum_{j \in S} \pi_{j}(\omega) d_{j}(\omega)\right] \\
\text { s.t. } \quad & \mathrm{E}\left[\gamma_{i}(\omega)\right] \leq c_{i} \quad i \in \cup_{j \in S} Z_{j} \\
& \pi_{j}(\omega)-\gamma_{i}(\omega) \leq s_{i j}, \quad j \in S, i \in \cup_{j \in S} Z_{j}, \omega \in \Omega \\
& \pi_{j}(\omega) \leq p_{j}, \quad j \in S, \omega \in \Omega \\
& \pi_{j}(\omega) \geq-h_{j}, \quad j \in S, \omega \in \Omega .
\end{aligned}
$$

We now provide some intuition on the dual. We assume that a company has to satisfy demands at the retailer stores. There are two alternative approaches to satisfy the demand. In the first approach, the company will place orders through the warehouses and allocate inventory to the retailer stores. The company would like to do this at a minimal cost. This is exactly the primal problem (1). In the second approach, the company outsources the ordering and allocation to another entity, which will charge the company depending on the realization of the underlying 
uncertainty. The entity would like to maximize the total charge. However, given the company's alternative approach, the company may impose certain constraints on the charge. Specifically, given the underlying primitive uncertainty $\omega$, the entity will charge a final unit price $\pi_{j}(\omega)$ for demand at retailer store $j$. This charge will compensate the unit price, $\gamma_{i}(\omega)$, charged by warehouse $i$ if the demand is satisfied by the order from the warehouse plus the transportation cost. The company insists that the expected unit price for each warehouse should be no more than the unit ordering cost; otherwise the company has incentive to place the order itself. This gives the first constraint. The second constraint implies that the final unit price charged should be no more than the unit price imposed by the corresponding warehouse plus the transportation cost. The third constraint implies that the final unit price should be no more than the lost sales penalty cost; otherwise the company is better off by ordering nothing and paying lost sales penalty. The last constraint implies that the final unit price should be no less than the negative inventory holding cost (i.e., the compensation to the company should be no more than the inventory holding cost); otherwise the company would be better off by reporting a higher demand to the other entity and holding inventory.

The following result is an immediate consequence of Theorem 1.

Corollary 1. For any collection of retailers $S \subseteq N, C(S)$ is equal to the optimal value of (4).

Proof. Given $\mathrm{E}\left[d_{j}(\omega)\right]<\infty$ for any $j \in N$, it is straightforward to verify that problem (1) satisfies the conditions of Theorem 1.

\subsection{Non-emptiness of the core}

Now we are ready to prove our first result. Denote by $(\pi(\omega), \gamma(\omega))$ an optimal solution of (Dual) $S$ with $S=N$. Then define

$$
l_{j}=\mathrm{E}\left[\pi_{j}(\omega) d_{j}(\omega)\right]
$$

Theorem 2. The vector $l=\left(l_{1}, \cdots, l_{N}\right)$ defined by (5) is an allocation in the core of the cooperative inventory game $(N, C)$. 
Proof. By definition,

$$
\sum_{j \in N} l_{j}=\sum_{j \in N} \mathrm{E}\left[\pi_{j}(\omega) d_{j}(\omega)\right]
$$

From Corollary 1 and the fact that $(\pi(\omega), \gamma(\omega))$ is the optimal solution of (Dual) ${ }_{N}$, we know that

$$
\sum_{j \in N} \mathrm{E}\left[\pi_{j}(\omega) d_{j}(\omega)\right]=C(N)
$$

Therefore, $l=\left(l_{1}, \cdots, l_{N}\right)$ is an allocation of the cooperative game $(N, C)$.

On the other hand, since $(\pi(\omega), \gamma(\omega))$ is a feasible solution to (Dual) $)_{N}$, then $(\pi(\omega), \gamma(\omega))$ (actually by restricting it to the set $S$ ) gives us a feasible solution to (Dual) $)_{S}$, for any $S \subseteq N$.

The objective function of (Dual) $)_{S}$ corresponding to the feasible solution $\pi_{j}(\omega)$ is

$$
\mathrm{E}\left[\sum_{j \in S} \pi_{j}(\omega) d_{j}(\omega)\right]=\sum_{j \in S} \mathrm{E}\left[\pi_{j}(\omega) d_{j}(\omega)\right]=\sum_{j \in S} l_{j} .
$$

Recall that, by Corollary $1, C(S)$ is equal to the optimal value of (Dual) $)_{S}$. Therefore,

$$
\sum_{j \in S} l_{j} \leq C(S)
$$

which shows that the allocation $l=\left(l_{1}, \cdots, l_{N}\right)$ is in the core.

Theorem 2 suggests a way to compute an allocation in the core. In particular, if there is only finite number of scenarios, the allocation can be easily computed by solving a linear program.

In the following example, we show that the core may be larger than the set of vectors derived from the optimal dual prices.

Example 1. Consider an example where there is only one warehouse 1 and two retailers 1 and 2 . The cost parameter is given by

$$
c_{1}=5 ; p_{1}=p_{2}=10 ; h_{1}=h_{2}=2,
$$

and the transportation costs are all equal to 0 . The random demands that the two retailers face are specified by a set $\Omega=\left\{\omega_{1}, \omega_{2}, \omega_{3}\right\}$ so that $\omega_{1}$ happens with probability $.3, \omega_{2}$ happens with probability .5 , and $\omega_{3}$ happens with probability .2. Further,

$$
\left(d_{1}\left(\omega_{k}\right), d_{2}\left(\omega_{k}\right)\right)= \begin{cases}(2,1), & \text { if } k=1 \\ (1,3), & \text { if } k=2 \\ (5,5), & \text { if } k=3\end{cases}
$$


In this case, it is easy to verify that $C(\{1,2\})=32.6, C(\{1\})=16$, and $C(\{2\})=20.2$. Also, $\left(\pi\left(\omega_{k}\right): k=1,2,3\right)=(-2,7.2,10)$ is the unique dual optimal solution. This optimal dual solution defines a unique vector $l=(12.4,20.2)$ that is in the core. (Please refer to the Appendix for the details.)

However, by definition, the core of this game is given by the set

$$
\left\{\left(l_{1}, l_{2}\right) \mid l_{1}+l_{2}=32.6, l_{1} \leq 16, l_{2} \leq 20.2\right\} .
$$

Therefore, the allocation $(12.4,20.2)$ defined by the optimal dual solution is a true subset of the core.

\subsection{A Special Case: Newsvendor Game}

In this subsection, we consider a special case of (1), the newsvendor problem. We show that the dual of the newsvendor problem has a closed form solution under very general conditions. In this case, we drop the subscript of all the cost parameters, and assume there is only one retailer who is facing a random and non-negative demand $d(\omega)$. Let $F(\cdot)$ be the cumulative distribution of $d(\omega)$. For any $v>0$, define

$$
F_{-}(v)=\lim _{m \rightarrow \infty} F\left(v-\frac{1}{m}\right)
$$

let $F_{-}(0)=0$. Observe that $F(\cdot)$ is non-decreasing and right continuous, the limit in $(6)$ is always attained.

Now the primal of the newsvendor problem becomes

$$
\begin{array}{cl}
\text { (Linear-P) } & \min \quad G(y):=c y+\mathrm{E}[Q(y, d(\omega))] \\
& \text { s.t. } \quad y \geq 0
\end{array}
$$

where

$$
Q(y, d(\omega))=p(d(\omega)-y)^{+}+h(y-d(\omega))^{+}
$$

with $\sigma^{+}=\max \{\sigma, 0\}$.

Note that the sub-differential set of $Q(y, d(\omega))$ with respect to $y$ is given as follows.

$$
\partial Q_{y}(y, d(\omega))= \begin{cases}h & \text { if } d(\omega)<y \\ {[-p, h]} & \text { if } d(\omega)=y \\ -p & \text { otherwise }\end{cases}
$$


According to Birge and Louveaux [3] Chapter 3, Corollary 12, $\lambda$ is an optimal solution to the above problem if and only if there exists $\mu \geq 0$ with $\lambda \mu=0$ such that

$$
\mu \in c+E\left[\partial Q_{y}(\lambda, d(\omega))\right]=\left[(p+h) F_{-}(\lambda)-(p-c),(p+h) F(\lambda)-(p-c)\right]
$$

which immediately implies that the following result.

Lemma 1. For any optimal solution $\lambda, F(\lambda) \geq \frac{p-c}{p+h}$. Moreover, if $\lambda>0$, then $F_{-}(\lambda) \leq \frac{p-c}{p+h} \leq F(\lambda)$.

The dual of (Linear-P) is very simple:

$$
\begin{array}{lll}
\text { (Linear-D) } & \max & \mathrm{E}[d(\omega) \pi(\omega)] \\
& \text { s.t. } & \mathrm{E}[\pi(\omega)] \leq c \\
& & -h \leq \pi(\omega) \leq p .
\end{array}
$$

In the following, we show that problem (Linear-D) has a closed form solution.

Theorem 3. Assume $d(\omega) \geq 0$. Let $\lambda$ be any optimal solution to (Linear-P) and let

$$
\pi(\omega)= \begin{cases}-h & \text { if } d(\omega)<\lambda \\ p-\eta & \text { if } d(\omega)=\lambda \\ p & \text { otherwise }\end{cases}
$$

where

$$
\eta= \begin{cases}\in[0, p+h] & \text { if } F(\lambda)=F_{-}(\lambda) \\ \left(\frac{p-c-(p+h) F_{-}(\lambda)}{F(\lambda)-F_{-}(\lambda)}\right)^{+} & \text {if } F(\lambda)>F_{-}(\lambda) .\end{cases}
$$

Then $\pi(\omega)$ is an optimal solution to problem (Linear-D).

The proof follows from the economic interpretation of the optimal dual variable $\pi(\omega)$. Indeed, since an optimal dual variable $\pi(\omega)$ is the marginal cost (or shadow price) per unit increase of the demand $d(\omega)$, it must be an element in the sub-differential set of $Q(y, d(\omega))$ with respect to $d(\omega)$, i.e.,

$$
\pi(\omega) \in \partial Q_{d}(y, d(\omega))=-\partial Q_{y}(y, d(\omega))
$$

In fact, one can verify that the optimal dual solutions are precisely those sub-gradients in the sub-differential set that satisfy the optimality conditions, which is exactly given by Theorem 3 . 


\section{Inventory Games with Concave Ordering Cost}

In this section, we consider another aspect that could motivate the retailers to cooperate: the ordering cost is a concave function of the order quantity. This cost structure is widely used in practice. For instance, it arises when suppliers provide incremental discount to additional units beyond some threshold.

The inventory game that we consider in this section is exactly the same as the one described in Subsection 2.2, except that we assume the ordering cost of retailer $i$, denoted by $c_{i}(\cdot)$, is a concave function of the order quantity. For ease of presentation, we assume that $c_{i}(\cdot)$ is continuously differentiable. Also, we assume that the demand of each retailer is non-negative. For any coalition $S$, the characteristic cost function $C(S)$ is defined by

$$
C(S)=\min _{y_{i} \geq 0, i \in \cup_{j \in S Z_{j}}} \sum_{i \in \cup_{j \in S} Z_{j}} c_{i}\left(y_{i}\right)+\mathrm{E}\left[f\left(y, d^{S}(\omega)\right],\right.
$$

where $f\left(y, d^{S}\right)$ is given in (1).

\subsection{A General Result}

In order to study the cooperative game with the characteristic function $C(S)$ by (8), we consider the duality result concerning the following two-stage stochastic program that generalizes (2):

$$
o^{*}:=\min _{x_{1} \in \Lambda} \nu_{1}\left(x_{1}\right)+\mathrm{E}\left[g\left(x_{1}, \omega\right)\right]
$$

where $\nu_{1}\left(x_{1}\right)$ is a concave function with $\nu_{1}(0)=0$,

$$
\Lambda=\left\{x_{1}: A_{11} x_{1}=b_{1}, x_{1} \geq 0\right\}
$$

and

$$
\begin{array}{ll}
g\left(x_{1}, \omega\right)=\min & \nu_{2}^{T} x_{2} \\
\text { s.t. } & A_{12} x_{1}+A_{22} x_{2}=b_{2}(\omega) \\
& x_{2} \geq 0 .
\end{array}
$$

It follows directly from the concavity of $\nu_{1}\left(x_{1}\right)$ that,

$$
o^{*}=\min _{x_{1} \in \Lambda} \min _{y_{1} \in \Lambda} \nu_{1}\left(x_{1}\right)-\left(\nabla \nu_{1}\left(x_{1}\right)\right)^{T} x_{1}+\left(\nabla \nu_{1}\left(x_{1}\right)\right)^{T} y_{1}+\mathrm{E}\left[g\left(y_{1}, \omega\right)\right]
$$


where $\nabla \nu_{1}(\cdot)$ is a sub-gradient of function $\nu_{1}(\cdot)$. Therefore, we obtain immediately that

$$
\begin{aligned}
o^{*}= & \max \quad o \\
& \text { s.t. } \quad o \leq \nu_{1}\left(x_{1}\right)-\left(\nabla \nu_{1}\left(x_{1}\right)\right)^{T} x_{1}+\min _{y_{1} \in \Lambda}\left(\nabla \nu_{1}\left(x_{1}\right)\right)^{T} y_{1}+\mathrm{E}\left[g\left(y_{1}, \omega\right)\right], \quad \forall x_{1} \in \Lambda .
\end{aligned}
$$

However, notice that for any given $x_{1}$, the minimization problem

$$
\min _{y_{1} \in \Lambda}\left(\nabla \nu_{1}\left(x_{1}\right)\right)^{T} y_{1}+\mathrm{E}\left[g\left(y_{1}, \omega\right)\right]
$$

is a two-stage linear program whose dual is

$$
\begin{array}{ll}
\max & \mathrm{E}\left[b_{1}^{T} \xi_{1}+b_{2}^{T}(\omega) \xi_{2}(\omega)\right] \\
\text { s.t. } & A_{11}^{T} \xi_{1}+\mathrm{E}\left[A_{12}^{T} \xi_{2}(\omega)\right] \leq \nabla \nu_{1}\left(x_{1}\right) \\
& A_{22}^{T} \xi_{2}(\omega) \leq \nu_{2} .
\end{array}
$$

Thus, we have

$$
\begin{aligned}
o^{*}=\max & o \\
\text { s.t. } \quad & o \leq \nu_{1}\left(x_{1}\right)-\left(\nabla \nu_{1}\left(x_{1}\right)\right)^{T} x_{1}+\mathrm{E}\left[b_{1}^{T} \xi_{1}\left(x_{1}\right)+b_{2}^{T}(\omega) \xi_{2}\left(x_{1}, \omega\right)\right], \quad \forall x_{1} \in \Lambda \\
& A_{11}^{T} \xi_{1}\left(x_{1}\right)+\mathrm{E}\left[A_{12}^{T} \xi_{2}\left(x_{1}, \omega\right)\right] \leq \nabla \nu_{1}\left(x_{1}\right), \quad \forall x_{1} \in \Lambda \\
& A_{22}^{T} \xi_{2}\left(x_{1}, \omega\right) \leq \nu_{2}, \quad \forall x_{1} \in \Lambda .
\end{aligned}
$$

Furthermore, for any given $x_{1}$, one can define variables $\varrho_{1}\left(x_{1}\right)$ and $\varrho_{2}\left(x_{1}, \omega\right)$ so that

$$
\nu_{1}\left(x_{1}\right)-\left(\nabla \nu_{1}\left(x_{1}\right)\right)^{T} x_{1} \geq \mathrm{E}\left[b_{1}^{T} \varrho_{1}\left(x_{1}\right)+b_{2}^{T}(\omega) \varrho_{2}\left(x_{1}, \omega\right)\right] .
$$

We further define $\pi_{1}$ and $\pi_{2}(\omega)$ so that $\pi_{1} \leq \varrho_{1}\left(x_{1}\right)+\xi_{1}\left(x_{1}\right)$ and $\pi_{2}(\omega) \leq \varrho_{2}\left(x_{1}, \omega\right)+\xi_{2}\left(x_{1}, \omega\right)$, for any $x_{1} \in \Lambda$. It can be easily verified that

$$
\begin{aligned}
o^{*}=\max & \mathrm{E}\left[b_{1}^{T} \pi_{1}+b_{2}^{T}(\omega) \pi_{2}(\omega)\right] \\
\text { s.t. } \quad & \pi_{1} \leq \varrho_{1}\left(x_{1}\right)+\xi_{1}\left(x_{1}\right), \quad \forall x_{1} \in \Lambda \\
& \pi_{2}(\omega) \leq \varrho_{2}\left(x_{1}, \omega\right)+\xi_{2}\left(x_{1}, \omega\right), \quad \forall x_{1} \in \Lambda \\
& \mathrm{E}\left[b_{1}^{T} \varrho_{1}\left(x_{1}\right)+b_{2}^{T}(\omega) \varrho_{2}\left(x_{1}, \omega\right)\right] \leq \nu_{1}\left(x_{1}\right)-\left(\nabla \nu_{1}\left(x_{1}\right)\right)^{T} x_{1}, \quad \forall x_{1} \in \Lambda \\
& A_{11}^{T} \xi_{1}\left(x_{1}\right)+\mathrm{E}\left[A_{12}^{T} \xi_{2}\left(x_{1}, \omega\right)\right] \leq \nabla \nu_{1}\left(x_{1}\right), \quad \forall x_{1} \in \Lambda \\
& A_{22}^{T} \xi_{2}\left(x_{1}, \omega\right) \leq \nu_{2}, \quad \forall x_{1} \in \Lambda .
\end{aligned}
$$


Now we focus on the inventory model. Notice that problem (8) is a special case of problem (9). From the strong duality of problem (9), we obtain,

$$
\begin{aligned}
C(S)=\max & \mathrm{E}\left[\sum_{j \in S} d_{j}(\omega) \pi_{j}(\omega)\right] \\
\text { s.t. } \quad & \pi_{j}(\omega) \leq \varrho_{j}(x, \omega)+\xi_{j}(x, \omega), \quad \forall x \geq 0, j \in S \\
& \mathrm{E}\left[\sum_{j \in S} d_{j}(\omega) \varrho_{j}(x, \omega)\right] \leq \sum_{i \in \cup_{j \in S} Z_{j}}\left(c_{i}\left(x_{i}\right)-c_{i}^{\prime}\left(x_{i}\right) x_{i}\right), \quad \forall x \geq 0 \\
& \mathrm{E}\left[\xi_{j}(x, \omega)\right] \leq s_{i j}+c_{i}^{\prime}\left(x_{i}\right), \quad \forall x \geq 0, j \in S, i \in \cup_{j \in S} Z_{j} \\
& -h_{j} \leq \xi_{j}(x, \omega) \leq p_{j}, \quad \forall x \geq 0, j \in S, \omega \in \Omega .
\end{aligned}
$$

This result can also be obtained by using the strong duality result for the so-called DC programming; see Toland [39].

The intuition of problem (12) is similar to the one for problem (4). The difference here is that for a concave ordering cost, if the order quantity of $i$ is $x_{i}$, one can think of the cost being decomposed to a proportional cost $c_{i}^{\prime}\left(x_{i}\right)$ and a fixed cost $c_{i}\left(x_{i}\right)-c^{\prime}\left(x_{i}\right) x_{i}$. Thus, in addition to the charge $\xi(x, \omega)$ to compensate the proportional cost and the transportation cost, a charge $\varrho_{j}(x, \omega)$ to each unit demand of retailer $j$ will be incurred to compensate for the fixed cost.

Denote by $(\pi(\omega), \varrho(x, \omega), \xi(x, \omega))$ an optimal solution to problem (12) with $S=N$. Now we are ready to present the main result of this subsection. The proof of the following theorem is similar to that of Theorem 2.

Theorem 4. Define $l_{j}=\mathrm{E}\left[\pi_{j}(\omega) d_{j}(\omega)\right], j \in N$. Then the vector $\left(l_{1}, \cdots, l_{n}\right)$ is an allocation in the core of the inventory game $(N, C)$ with concave ordering cost, if $\varrho(x, \omega) \geq 0$ for any $x \geq 0$ and any $\omega \in \Omega$.

Theorem 4 requires the condition that $\varrho(x, \omega) \geq 0$ for any $x \geq 0$ and any $\omega \in \Omega$. We would like to point out that this condition is only a sufficient condition. It guarantees that any optimal dual solution to the grand coalition $N$ is feasible to the dual problem of coalition $S \subseteq N$.

However, this sufficient condition does not hold in general. In fact, we can show that the core of the inventory game with concave ordering cost could be empty. Indeed, consider the deterministic demand case. Assume that only a fixed cost is incurred at each warehouse if it orders; otherwise the ordering cost is zero. Under this assumption, the inventory game reduces to the so-called facility location game studied by Goemans and Skutella [11]. It is shown in [11] that the core of the facility location game could be empty. 
Nonetheless, the sufficient condition in Theorem 4 may hold in some cases. In the next subsection, we show that the condition holds for the newsvendor game with concave ordering cost.

\subsection{Newsvendor Game with Concave Ordering Cost}

Recall that the newsvendor game assumes that all retailers (newsvendors) have the same cost structure and the transhipment cost is zero. In the following, we denote by $d(\omega)$ the sum of individual retailers's demand.

In this case, the minimization problem of the grand coalition is

$$
\begin{aligned}
\text { (Concave-P) } & \min \quad c(x)+g(x) \\
& \text { s.t. } \quad x \geq 0
\end{aligned}
$$

where $c(x)$ is a concave function of $x$ with $c(0)=0$, and

$$
g(x)=h \mathrm{E}\left[(x-d(\omega))^{+}\right]+p \mathrm{E}\left[(d(\omega)-x)^{+}\right] .
$$

It is clear that $g(\cdot)$ is a convex function of $x$.

For ease of presentation, we assume $c(x)$ is continuously differentiable. However, all our results still hold when $c(x)$ is a general concave function with $c(0)=0$. For the rest of this section, we assume that $x^{*}$ is an optimal solution to problem (Concave-P).

It is clear that problem (Concave-P) is a special case of problem (8). Therefore, we can derive the dual of problem (Concave-P) as follows.

$$
\begin{array}{ll}
\text { (Concave-D) } \max \quad & \mathrm{E}[d(\omega) \pi(\omega)] \\
\text { s.t. } \quad & \pi(\omega) \leq l(x, \omega)+v(x, \omega), \quad \text { for all } x \geq 0, \omega \in \Omega \\
& \mathrm{E}[l(x, \omega) d(\omega)] \leq c(x)-c^{\prime}(x) x, \quad \text { for all } x \geq 0 \\
& \mathrm{E}[v(x, \omega)] \leq c^{\prime}(x), \quad \text { for all } x \geq 0 \\
& -h \leq v(x, \omega) \leq p, \quad \text { for all } x \geq 0, \omega \in \Omega .
\end{array}
$$

The variables $v(x, \omega)$ and $l(x, \omega)$ in (Concave-D) correspond to the variables $\xi(x, \omega)$ and $\varrho(x, \omega)$ in problem (12). 
Our main result of this subsection is to construct an optimal closed-form solution to (ConcaveD) such that $l(x, \omega) \geq 0$ for any $x \geq 0$ and any $\omega \in \Omega$. By Theorem 4 , this leads to a core allocation to the newsvendor game with concave ordering cost.

The construction of the dual solution relies on the property of the following problem defined for every fixed $x \geq 0$ :

$$
\begin{array}{cl}
\left(\mathbf{C P L}_{x}\right) & \min \quad c^{\prime}(x) y+g(y) \\
\text { s.t. } & y \geq 0
\end{array}
$$

and its dual

$$
\begin{array}{rll}
\left(\mathbf{C D L}_{x}\right) & \max & \mathrm{E}[v(x, \omega) d(\omega)] \\
\text { s.t. } & \mathrm{E}[v(x, \omega)] \leq c^{\prime}(x) \\
& -h \leq v(x, \omega) \leq p .
\end{array}
$$

By the concavity of $c(x)$, we know that

Lemma 2. Any optimal solution $x^{*}$ to problem (Concave-P) is optimal to the problem $\left(\mathbf{C P} \mathbf{L}_{x^{*}}\right)$.

The following two lemmas are also useful in the construction of the dual solution. We present the proofs in the Appendix.

Lemma 3. For any $x \geq 0$,

$$
(p+h) \mathrm{E}[d(\omega) \delta(d(\omega) \in[0, x])]=p \mathrm{E}[d(\omega)]-g(x)-x(p-(p+h) F(x)) .
$$

\section{Lemma 4.}

$$
(p+h) \mathrm{E}\left[d(\omega) \delta\left(d(\omega) \in\left[0, x^{*}\right]\right)\right]+x^{*}\left(p-(p+h) F\left(x^{*}\right)\right) \geq c\left(x^{*}\right)
$$

Now we define

$$
q^{*}:=\inf \left\{q:(p+h) \mathrm{E}\left[d(\omega) \delta\left(d(\omega) \in\left[q, x^{*}\right]\right)\right]+x^{*}\left(p-(p+h) F\left(x^{*}\right)\right) \leq c\left(x^{*}\right)\right\}
$$

The existence of $q^{*} \in\left[0, x^{*}\right]$ is implied by Lemma 4 and by the facts that $p-c^{\prime}\left(x^{*}\right)-(p+h) F\left(x^{*}\right) \leq 0$ (which follows from Lemmas 2 and 1 ) and $c\left(x^{*}\right)-c^{\prime}\left(x^{*}\right) x^{*} \geq 0$. Also, it is easy to verify that, if $q^{*}=0$ or if $F(\cdot)$ is continuous at $q^{*}$, i.e, $F\left(q^{*}\right)=F_{-}\left(q^{*}\right)$, then we must have

$$
(p+h) \mathrm{E}\left[d(\omega) \delta\left(d(\omega) \in\left[q^{*}, x^{*}\right]\right)\right]+x^{*}\left(p-(p+h) F\left(x^{*}\right)\right)=c\left(x^{*}\right)
$$


We are now ready to construct the dual optimal solution. Let $\lambda(x)$ be any optimal solution for problem $\left(\mathbf{C P L}_{x}\right)$ for $x \neq x^{*}$, and let $\lambda\left(x^{*}\right)=x^{*}$, which is an optimal solution to $\left(\mathbf{C P L}_{x^{*}}\right)$. For each $x \geq 0$ and $\omega$, define

$$
\begin{gathered}
v^{*}(x, \omega)= \begin{cases}-h & \text { if } d(\omega)<\lambda(x) \\
p-\eta(x) & \text { if } d(\omega)=\lambda(x) \\
p & \text { otherwise, }\end{cases} \\
\pi^{*}(\omega)= \begin{cases}p, & \text { if } d(\omega)>q^{*} ; \\
\rho^{*}-h, & \text { if } d(\omega)=q^{*} ; \\
-h, & \text { otherwise, }\end{cases}
\end{gathered}
$$

and

$$
l^{*}(x, \omega)=\left(\pi^{*}(\omega)-v^{*}(x, \omega)\right)^{+},
$$

where

$$
\eta(x)= \begin{cases}0 & \text { if } F(\lambda(x))=F_{-}(\lambda(x)) \\ \left(\frac{p-c^{\prime}(x)-(p+h) F_{-}(\lambda(x))}{F(\lambda(x))-F_{-}(\lambda(x))}\right)^{+} & \text {if } F(\lambda(x))>F_{-}(\lambda(x)),\end{cases}
$$

and

$\rho^{*}= \begin{cases}0 & \text { if }\left(F\left(q^{*}\right)-F_{-}\left(q^{*}\right)\right) q^{*}=0 \\ \frac{c\left(x^{*}\right)-(p+h) \mathrm{E}\left[d(\omega) \delta\left(d(\omega) \in\left(q^{*}, x^{*}\right]\right)\right]-x^{*}\left(p-(p+h) F\left(x^{*}\right)\right)}{\left(F\left(q^{*}\right)-F_{-}\left(q^{*}\right)\right) q^{*}}, & \text { otherwise. }\end{cases}$

It is clear from the construction that $l^{*}(x, \omega) \geq 0$ for any $x \geq 0$ and any $\omega \in \Omega$. Now we are ready to prove our main result of this subsection.

Theorem 5. $\left(\pi^{*}, v^{*}, l^{*}\right)$ defined above is an optimal solution to (Concave-D).

This result then directly implies that the newsvendor game with concave ordering cost has a non-empty core. More specifically, let $\left(\pi^{*}, v^{*}, l^{*}\right)$ be optimal to (Concave-D) with $l^{*} \geq 0$ and $d(\omega)=\sum_{j \in N} d_{j}(\omega)$. Define

$$
l_{j}=\mathrm{E}\left[\pi_{j}^{*}(\omega) d_{j}(\omega)\right]
$$

Theorem 6. Then vector $l=\left(l_{1}, \cdots, l_{N}\right)$ is an allocation in the core of the newsvendor game with concave ordering cost. 
Theorem 6 is based on the assumption that the concave ordering cost function is continuously differentiable. This differentiability assumption is only made for ease of presentation and can be relaxed.

We would like to point out that, although (Concave-D) is an infinite dimension stochastic linear program, it admits a closed-form solution. Therefore, we do not have to use algorithms designed for general infinite dimension stochastic linear program to solve (Concave-D). This observation is illustrated through the following example.

Example 2. Consider an example where there are three retailers 1,2 and 3 . The cost parameter is given by

$$
p=5, h=1, c(x)=\left\{\begin{array}{l}
3 x, \quad \text { if } 0 \leq x<6, \\
6+2 x, \quad \text { if } x \geq 6 .
\end{array}\right.
$$

The random demands that the three retailers face are specified by a set $\Omega=\left\{\omega_{1}, \omega_{2}, \omega_{3}\right\}$ so that $\omega_{1}$ happens with probability $.2, \omega_{2}$ happens with probability .4 , and $\omega_{3}$ happens with probability .4. Further,

$$
\left(d_{1}\left(\omega_{k}\right), d_{2}\left(\omega_{k}\right), d_{3}\left(\omega_{k}\right)\right)= \begin{cases}(4,3,3), & \text { if } k=1 \\ (4,4,4), & \text { if } k=2 \\ (4,6,4), & \text { if } k=3\end{cases}
$$

In this case, it is easy to verify that $C(\{1,2,3\})=34.4, C(\{1,2\})=26.2, C(\{1,3\})=22.2$, $C(\{2,3\})=26.4, C(\{1\})=12, C(\{2\})=16.2$, and $C(\{3\})=12.2$.

Now consider the dual problem of the grand coalition with aggregated demand. The optimal ordering quantity is $x^{*}=12$, which can be easily computed. Furthermore, recall the definition of $q^{*}:$

$$
q^{*}:=\inf \left\{q:(p+h) \mathrm{E}\left[d(\omega) \delta\left(d(\omega) \in\left[q, x^{*}\right]\right)\right]+x^{*}\left(p-(p+h) F\left(x^{*}\right)\right) \leq c\left(x^{*}\right)\right\} .
$$

Notice that $c\left(x^{*}\right)=6+12 \cdot 2=30$, and $F\left(x^{*}\right)=0.6$, It follows that

$$
(p+h) \mathrm{E}\left[d(\omega) \delta\left(d(\omega) \in\left[q, x^{*}\right]\right)\right]+x^{*}\left(p-(p+h) F\left(x^{*}\right)\right) \leq c\left(x^{*}\right)
$$

if and only if $q>x^{*}$. Therefore, $q^{*}=x^{*}=12$. Then from the definition of $\rho^{*}$, we get $\rho^{*}=11 / 4$. Thus, by construction, the optimal dual solution is given by

$$
\left(\pi^{*}\left(\omega_{1}\right), \pi^{*}\left(\omega_{2}\right), \pi^{*}\left(\omega_{3}\right)\right)=(-1,7 / 4,5)
$$


Now, the cost allocation defined by (21) is given by

$$
\begin{aligned}
& l_{1}=0.2 \cdot(-1) \cdot 4+0.4 \cdot(7 / 4) \cdot 4+0.4 \cdot 5 \cdot 4=10 \\
& l_{2}=0.2 \cdot(-1) \cdot 3+0.4 \cdot(7 / 4) \cdot 4+0.4 \cdot 5 \cdot 6=14.2 \\
& l_{3}=0.2 \cdot(-1) \cdot 3+0.4 \cdot(7 / 4) \cdot 4+0.4 \cdot 5 \cdot 4=10.2 .
\end{aligned}
$$

One can easily verify that this allocation is indeed in the core.

\section{$5 \quad$ Testing Membership of the Core}

Another important question concerning the inventory game is to check whether a given allocation is in the core or not. In this section, we show that even for a very simple case, the problem is NP-hard. More formally, we consider the following problem

\section{Problem TMOC}

Instance: A cooperative newsvendor game $(N, C)$ and a vector $l=\left(l_{1}, \cdots, l_{n}\right)$ with $\sum_{j \in N} l_{j}=C(N)$.

Question: Is $l$ not an element of the core of the game, i.e., does there exist a coalition $S \subseteq N$ such that $\sum_{j \in S} l_{j}>C(S) ?$

Theorem 7. Problem TMOC is NP-hard.

Proof. To prove the hardness, we establish a polynomial transformation from the Subset Sum problem to the TMOC. The Subset Sum problem is a well-known NP-complete problem which can be described as follows:

\section{Subset Sum}

Instance: A set of integers $\left\{a_{1}, a_{2}, \cdots, a_{n}\right\}$.

Question: Is there a subset $S \subseteq\{1,2, \cdots, n\}$ such that $\sum_{j \in S} a_{j}=0$ ?

Given an instance of the Subset Sum problem, we construct an instance of the TMOC as follows. There are $n+1$ retailers, i.e., $N=\{1,2, \cdots, n+1\}$. Let the vector $l=\left(\frac{1}{n+1}, \cdots, \frac{1}{n+1}\right)$. 
Let the ordering $\operatorname{cost} c=0$, the inventory holding cost and penalty cost are equal to 2, i.e., $p=h=2$. The random demand is given by

$$
d(\omega)=\left(d_{1}(\omega), \cdots, d_{n}(\omega), d_{n+1}(\omega)\right)=\left\{\begin{array}{l}
\left(d_{1}^{1}, d_{2}^{1}, \cdots, d_{n}^{1}, d_{n+1}^{1}\right), \text { with probability }=.5 \\
\left(d_{1}^{2}, d_{2}^{2}, \cdots, d_{n}^{2}, d_{n+1}^{2}\right), \text { with probability }=.5
\end{array}\right.
$$

such that $d_{j}^{1}, d_{j}^{2}$ are nonnegative, and $d_{j}^{1}-d_{j}^{2}=2 a_{j}$ for each $j=1,2, \cdots, n$, and $d_{n+1}^{1}-d_{n+1}^{2}=$ $1-2 \sum_{j=1}^{n} a_{j}$.

Let $\bar{a}_{j}=2 a_{j}$ for $j=1,2, \cdots, n$, and $\bar{a}_{n+1}=1-2 \sum_{j=1}^{n} a_{j}$. Then

$$
\sum_{j=1}^{n+1} \bar{a}_{j}=1
$$

Therefore, for any $S \subseteq N$,

$$
\begin{aligned}
C(S) & =\min _{y \geq 0} \mathrm{E}\left[2\left|\sum_{j \in S} d_{j}(\omega)-y\right|\right] \\
& =\min _{y \geq 0}\left|\sum_{j \in S} d_{j}^{1}-y\right|+\left|\sum_{j \in S} d_{j}^{2}-y\right| \\
& =\left|\sum_{j \in S} d_{j}^{1}-\sum_{j \in S} d_{j}^{2}\right| \\
& =\left|\sum_{j \in S} \bar{a}_{j}\right|
\end{aligned}
$$

Then

$$
C(N)=\left|\sum_{j \in N} \bar{a}_{j}\right|=1=\sum_{j \in N} l_{j}
$$

which implies that $l$ is an allocation. Now we show that the allocation $l=\left(\frac{1}{n+1}, \cdots, \frac{1}{n+1}\right)$ is not in the core of the newsvendor game if and only if there exists a subset $S \subseteq\{1,2, \cdots, n\}$ such that $\sum_{j \in S} a_{j}=0$. Then the NP-hardness of problem TMOC follows immediately.

Case 1. If $l$ is not in the core, then there exists an $T \subset N$ such that

$$
C(T)<\sum_{j \in T} l_{j} \leq 1
$$

and hence $C(T)=0$. This is because $C(T)=\left|\sum_{j \in T} \bar{a}_{j}\right|$ and all $a_{j}$ are integers, which implies that $C(T)$ is also an (non-negative) integer. Therefore,

$$
\sum_{j \in T} \bar{a}_{j}=0 .
$$


We claim that $n+1 \notin T$ because all $\bar{a}_{j}=2 a_{j}, j=1,2, \cdots, n$, are even, while $\bar{a}_{n+1}$ is odd. Therefore, $T \subseteq\{1,2, \cdots, n\}$. Then

$$
\sum_{j \in T} a_{j}=\frac{1}{2} \sum_{j \in T} \bar{a}_{j}=0 .
$$

Case 2. If $l$ is in the core, then for every $T \subseteq N, C(T) \geq \sum_{j \in T} l_{j}>0$. But for $S \subseteq$ $\{1,2, \cdots, n\} \subset N$,

$$
C(S)=\left|\sum_{j \in S} \bar{a}_{j}\right|=2\left|\sum_{j \in S} a_{j}\right| .
$$

Thus, $\sum_{j \in S} a_{j} \neq 0$ for all $S \subseteq\{1,2, \cdots, n\}$.

This completes the proof.

\section{Concluding Remarks}

In this paper, we demonstrated the power of stochastic programming duality approach in analyzing a broad class of stochastic inventory centralization games. Although we mainly focused on the model proposed by [28], the approach is readily applicable to more general models. Here we describe a few examples.

In a very recent paper [30], an inventory centralization game with delivery restrictions is studied. The new element of this game, compared to the one described in Subsection 2.2, is that each retailer $j$ is associated with a critical demand level $\epsilon_{j}$. If the realized demand of $j, d_{j}$, is lower than $\epsilon_{j}$, then only $d_{j}$ units need to be satisfied; otherwise, at least $\epsilon_{j}$ units of demand or retailer $j$ need to be satisfied. It is also required that before the demand realization, the total amount of goods ordered should be at least $\sum_{j \in S} \epsilon_{j}$ for any coalition $j$. In order to analyze this game, we can simply add one constraint

$$
\sum_{i \in \cup_{j \in S} Z_{j}} y_{i} \geq \sum_{j \in S} \epsilon_{j}
$$

to the first stage formulation of the stochastic linear program (1), and add another set of constraints

$$
\sum_{i \in \cup_{j \in S} Z_{j}} x_{i j} \geq \min \left\{\epsilon_{j}, d_{j}\right\} \quad j \in S
$$

to the second stage formulation. We can then write the dual of this new stochastic linear program. Using the duality approach, we can identify a core allocation of this cooperative game. We re- 
mark that the model in [30] is actually slightly simpler than what we described above in that the transportation cost of allocating inventory was ignored.

In another paper [31], Ozen, Sošić, and Slikker propose an interesting three-stage inventory centralization game. At the time orders are placed by the warehouses, the demand distributions of retailers are known, but not the exact values of the demands. In the second stage, after orders arrived at warehouses, retailers observe demand signals and update their demand forecast. In the last stage, inventory are allocated to the retailers and the actual demands are realized. This inventory model can be formulated as a three-stage stochastic linear program. The same duality approach in this paper has been applied to identify a core allocation for the three-stage inventory centralization game. We should mention that [31] contains other interesting results as well.

The stochastic programming duality approach may also be applied to inventory centralization games with multiple products, where the demands for these products can be correlated.

Indeed, as the strong duality of linear programming has played an important role in studying deterministic cooperative games, we expect that the duality theory of stochastic linear programming may as well play a similar role in studying cooperative games with stochastic elements in other supply chain settings and beyond.

Acknowledgement. The authors wish to thank the associate editor and two referees for helpful comments that have helped improve this paper significantly. The associate editor and one of the referees suggested us to present the result in Subsection 4.2 within a more general framework, which results in the result in Subsection 4.1. They also helped us simplify the proofs Lemma 1 and Theorem 3. The authors also thank Eitan Zemel for helpful discussions on cooperative inventory games, and Roger Wets for kindly answering our questions on duality of stochastic linear programming.

\section{References}

[1] Anily, S. and Haviv, M. (2003). The Cost Allocation Problem for the First Order Interaction Joint Replenishment Model. Operations Research, Forthcoming.

[2] Anupindi, R., Bassok, Y. and Zemel, E. (2001). A General Framework for the Study of Decen- 
tralized Distribution Systems. Manufacturing 8 Service Operations Management, 3(4):349368.

[3] Birge, J. and Louveaux, F. (1997). Introduction to Stochastic Programming, Springer-Verlag, New York.

[4] Chen, X. and Zhang, J. (2006). Duality approaches to economic lot sizing games. Submitted.

[5] Deng, X. and Papadimitriou, C.H. (1994). On the complexity of cooperative solution concepts. Mathematics of Operations Research, 19: 257-266

[6] Dror, M. and Hartman, B. (2006). Shipment Consolidation: Who pays for it and how much. Management Science, In press.

[7] Faigle, U., W. Kern, S. P. Fekete and W. Hochstttler (1997). On the complexity of testing membership in the core of min-cost spanning tree games, Int. J. Game Theory 26: 361-366.

[8] Cooperation and Collaboration between Logistic Service Providors (LSP's): Threat or Opportunity? European Chemical Transport Association White Paper, 2006.

[9] Eppen, G.D. (1979). Effects of centralization on expected costs in a multi-location news- boy problem. Management Science, 25(5):498-501.

[10] Gerchak, Y. and Gupta, D. (1991). On apportioning costs to customers in central- ized continuous review inventory systems. Journal of the Operations Management, 10(4):546-551.

[11] Goemans, M.X. and Skutella, M. (2004). Cooperative facility location game. Journal of Algorithms, 50(2):194-214.

[12] Granot, D. (1986). A generalized linear production model: A unifying model. Mathematical Programming, 34(2):212-222.

[13] Granot, D. and Sošić, G. (2003). A Three Stage Model for a Decentralized Distribution System of Retailers. Operations Research, 51(5):771-784.

[14] Hartman, B. and Dror, M. (1996). Cost allocation in continuous review inventory models. Naval Research Logistics Quarterly, 43:549-561. 
[15] Hartman, B. and Dror, M. (1997). Inventory centralization costs with varying cost parameters. Working paper, MIS Department, University of Arizona.

[16] Hartman, B. and Dror, M. (2005). Allocation of gains from inventory centralization in newsvendor environments. IIE Transaction, 37:93-107.

[17] Hartman, B., Dror, M. and Shaked, M. (2000). Cores of inventory centralization games. Games and Economic Behavior, 31:26-49.

[18] Kleywegt, A.J. and Shapiro, A. (2001). Stochastic Optimization, Handbook of Industrial Engineering, 3rd Edition, Gavriel Salvendy, Ed., John Wiley, New York, pp. 2625-2650.

[19] Korf, L. (2004). Stochastic programming duality: $L^{\infty}$ multipliers for unbounded constraints with an application to mathematical finance. Mathematical Programming, 99:241-259.

[20] Linderoth, J., Shapiro, A., and Wright S. (2006). The empirical behavior of sampling methods for stochastic programming, Ann Oper Res, 142:215-241.

[21] Meca, A., Timmer, J., Garcia-Jurado, I., and Borm, P. (2004). Inventory games. European Journal of Operational Research, 156:127-139.

[22] Montrucchio, L. and Scarsini, M. (2007). Large newsvendor games. Games and Economic Behavior, 58(2):316-337.

[23] Müller, A., Scarsini, M. and Shaked, M. (2002). The newsvendor game has a non-empty core. Games and Economic Behavior, 38:118-126.

[24] Nagarajan, M. and Sošić, G. (2006). Stable Farsighted Coalitions in Competitive Markets. Management Science, to appear.

[25] Nagarajan, M. and Sošić, G. (2006). Game-Theoretical Analysis of Cooperation Among Supply Chain Agents: Review and Extensions. European Journal of Operational Research, to appear.

[26] Collaborative Commmerce: Complelling Benefits, Significant Obstacles. NerveWire Inc. White Paper, 2002.

[27] Owen, G. (1975). On the core of linear production games. Mathematical Programming, 9:358370. 
[28] Ozen, U., Fransoo, J., Norde, H. and Slikker, M. (2004). Cooperation between multiple newsvendors with warehouses. Working Paper, 2004.

[29] Ozen, U., Norde, H. and Slikker, M. (2005). On the convexity of newsvendor games. Beta working paper WP-131, Technische Universiteit Eindhoven, Eindhoven, The Netherlands and CentER Discussion Paper 2005-103, Tilburg University, Tilburg, The Netherlands.

[30] Ozen, U., Erkip, N., and Slikker, M. (2006). Profit allocation in newsvendor situations with delivery constraints. Beta working paper WP-186, Technische Universiteit Eindhoven, Eindhoven, The Netherlands.

[31] Ozen, U. and Sošić, G. (2007). A collaborative decentralized distribution system with demand updates. Working paper, University of Southern California.

[32] Rockafellar, R. (1970). Convex analysis, Princeton University Press, Princeton, N.J.

[33] Rockafellar, R. and Wets R. (1976). Stochastic convex programming: relatively complete recourse and induced feasibility. SIAM J. Control and Optimization, 14(3):574-589.

[34] Shapiro, A. and T. Homem-de-Mello. (1998). A Simulation-Based Approach to Stochastic Programming with Recourse. Mathematical Programming 81:301-325.

[35] Slikker, M., Fransoo, J., and Wouters, M. (2001). Joint ordering in multiple news-vendor situations: a game theoretical approach. BETA Working Paper, 64, Eindhoven University of Technology, Eindhoven, The Netherlands.

[36] Slikker, M., Fransoo, J., and Wouters, M. (2005). Cooperation between multiple news-vendors with transshipments. European Journal of Operational Research, 167(2):370-380.

[37] Strozniak, P. Collaborative logistics: overcoming its challenges can lower transportation and inventory costs and reduce stockouts - In depth report: logistics Frontline Solutions, August, 2003.

[38] Tamir, T. (1991). On the core of network synthesis games. Mathematical Programming, 50:123135.

[39] Toland, J. F. (1979). On subdifferential calculus and duality in non-convex optimization, Mémoirs de la S.M.F. 60 (1979), pp. 177-183. 
[40] Van den Heuvel, W., Borm, P. E.M., and Hamers, H. (2005). Economic Lot-Sizing Games. European Journal of Operational Research, to appear.

[41] Zhang, J. (2006). Cost Allocation for Joint Replenishment Models. Submitted.

\section{Appendix: Proofs}

\section{Example 1: Computation}

In order to compute $C(\{1,2\})$, we solve a newsvendor problem with the aggregated demand

$$
d\left(\omega_{k}\right)=d_{1}\left(\omega_{k}\right)+d_{2}\left(\omega_{k}\right)= \begin{cases}3, & \text { if } k=1 \\ 4, & \text { if } k=2 \\ 10, & \text { if } k=3\end{cases}
$$

In this case, the newsvendor problem is a linear program

$$
\begin{array}{ll}
\min & 5 x+0.3 \cdot\left(2 I_{1}+10 z_{1}\right)+0.5 \cdot\left(2 I_{2}+10 z_{2}\right)+0.2 \cdot\left(2 I_{3}+10 z_{3}\right) \\
\text { s.t. } & I_{1} \geq x-3, I_{2} \geq x-4, I_{3} \geq x-10 \\
& z_{1} \geq 3-x, z_{2} \geq 4-x, z_{3} \geq 10-x \\
& x, I_{k}, z_{k} \geq 0, k=1,2,3 .
\end{array}
$$

The dual of this linear program is

$$
\begin{array}{ll}
\max & 0.3 \cdot 3 \cdot \pi\left(\omega_{1}\right)+0.5 \cdot 4 \cdot \pi\left(\omega_{2}\right)+0.2 \cdot 10 \cdot \pi\left(\omega_{3}\right) \\
\text { s.t. } & 0.3 \cdot \pi\left(\omega_{1}\right)+0.5 \cdot \pi\left(\omega_{2}\right)+0.2 \cdot \pi\left(\omega_{3}\right) \leq 5 \\
& -2 \leq \pi\left(\omega_{k}\right) \leq 10, k=1,2,3 .
\end{array}
$$

The optimal values of both problems are 32.6 and an optimal primal solution is $x=4$. Furthermore, any optimal dual solution must satisfy

$$
0.3 \cdot \pi\left(\omega_{1}\right)+0.5 \cdot \pi\left(\omega_{2}\right)+0.2 \cdot \pi\left(\omega_{3}\right)=5
$$

and

$$
0.3 \cdot 3 \cdot \pi\left(\omega_{1}\right)+0.5 \cdot 4 \cdot \pi\left(\omega_{2}\right)+0.2 \cdot 10 \cdot \pi\left(\omega_{3}\right)=32.6 \text {. }
$$


It follows that

$$
0.5 \cdot \pi\left(\omega_{2}\right)+1.4 \cdot \pi\left(\omega_{3}\right)=17.6 .
$$

Therefore, if $\left(\pi_{1}\left(\omega_{k}\right): k=1,2,3\right)$ is an optimal basic feasible solution, we must have $\pi\left(\omega_{3}\right)=10$, $\pi\left(\omega_{2}\right)=7.6$, and then $\pi\left(\omega_{1}\right)=-2$. (If $\pi\left(\omega_{3}\right)=-2$, then from $0.5 \cdot \pi\left(\omega_{2}\right)+1.4 \cdot \pi\left(\omega_{3}\right)=17.6$ we know that $\pi\left(\omega_{2}\right)>10$, which is a contradiction). Then we conclude that $\left(\pi\left(\omega_{k}\right): k=1,2,3\right)=$ $(-2,7.2,10)$ is the only basic feasible solution that is optimal. But the feasible set of the dual problem is bounded. Thus, $\left(\pi\left(\omega_{k}\right): k=1,2,3\right)=(-2,7.2,10)$ is the unique dual optimal solution.

This optimal dual solution defines a unique vector $l=(12.4,20.2)$ that is in the core.

\section{Proof of Lemma 3}

By definition

$$
g(x)=h \mathrm{E}\left[(x-d(\omega))^{+}\right]+p \mathrm{E}\left[(d(\omega)-x)^{+}\right] .
$$

Notice that

$$
(d(\omega)-x)^{+}=d(\omega)-x+(x-d(\omega))^{+} .
$$

Therefore

$$
\begin{aligned}
g(x) & =(p+h) \mathrm{E}\left[(x-d(\omega))^{+}\right]+p \mathrm{E}[d(\omega)]-p x \\
& =(p+h) \mathrm{E}\left[(x-d(\omega))^{+} \delta(d(\omega) \in[0, x]]+p \mathrm{E}[d(\omega)]-p x\right. \\
& =(p+h) x \mathrm{E}[\delta(d(\omega) \in[0, x]]-(p+h) \mathrm{E}[d(\omega) \delta(d(\omega) \in[0, x]]+p \mathrm{E}[d(\omega)]-p x \\
& =(p+h) x F(x)-(p+h) \mathrm{E}[d(\omega) \delta(d(\omega) \in[0, x]]+p \mathrm{E}[d(\omega)]-p x .
\end{aligned}
$$

By rearranging the terms, Lemma 3 follows. 


\section{Proof of Lemma 4}

By Lemma 3, and the fact that $c(0)=0$, we get

$$
\begin{aligned}
& (p+h) \mathrm{E}\left[d(\omega) \delta\left(d(\omega) \in\left[0, x^{*}\right]\right)\right]+x^{*}\left(p-(p+h) F\left(x^{*}\right)\right) \\
= & p \mathrm{E}[d(\omega)]-g\left(x^{*}\right) \\
= & p \mathrm{E}[d(\omega)]-\left\{c\left(x^{*}\right)+g\left(x^{*}\right)\right\}+c\left(x^{*}\right) \\
\geq & p \mathrm{E}[d(\omega)]-\{c(0)+g(0)\}+c\left(x^{*}\right) \\
= & c\left(x^{*}\right),
\end{aligned}
$$

where the inequality follows from the fact that $x^{*}$ is an optimal solution to (Concave-P). The proof is complete.

\section{Proof of Theorem 5}

It is easy to verify that $\eta(x) \in[0, p+h]$ for any $x \geq 0$, and Lemma 4 implies that $\rho^{*} \in[0, p+h]$. Also, when $\left(F\left(q^{*}\right)-F_{-}\left(q^{*}\right)\right) q^{*}=0$, then (20) holds. Therefore, in any case, we have

$$
q^{*}\left(F\left(q^{*}\right)-F_{-}\left(q^{*}\right)\right) \rho^{*}=c\left(x^{*}\right)-(p+h) \mathrm{E}\left[d(\omega) \delta\left(d(\omega) \in\left(q^{*}, x^{*}\right]\right)\right]-x^{*}\left(p-(p+h) F\left(x^{*}\right)\right) .
$$

In order to prove Theorem 5, we first establish two lemmas based on the contraction of the dual solution.

Lemma 5. If $q^{*}=x^{*}$ and $\left(F\left(x^{*}\right)-F_{-}\left(x^{*}\right)\right) x^{*}>0$, then $\rho^{*}+\eta\left(x^{*}\right) \geq p+h$, where the equality holds only if $c\left(x^{*}\right)-c^{\prime}\left(x^{*}\right) x^{*}=0$.

Proof. By definition, if $q^{*}=x^{*}$ and $\left(F\left(x^{*}\right)-F_{-}\left(x^{*}\right)\right) x^{*}>0$, then

$$
\begin{aligned}
\rho^{*}+\eta\left(x^{*}\right) & \geq \frac{p-c^{\prime}\left(x^{*}\right)-(p+h) F_{-}\left(x^{*}\right)}{F\left(x^{*}\right)-F_{-}\left(x^{*}\right)}+\frac{c\left(x^{*}\right)-x^{*}\left(p-(p+h) F\left(x^{*}\right)\right)}{x^{*}\left(F\left(x^{*}\right)-F_{-}\left(x^{*}\right)\right)} \\
& =(p+h)+\frac{c\left(x^{*}\right)-c^{\prime}\left(x^{*}\right) x^{*}}{x^{*}\left(F\left(x^{*}\right)-F_{-}\left(x^{*}\right)\right)} \\
& \geq p+h .
\end{aligned}
$$

The lemma follows. 
Lemma 6. For any $x \geq 0, l^{*}(x, \omega)=0$ or

$$
\mathrm{E}\left[l^{*}(x, \omega) d(\omega)\right]=g\left(x^{*}\right)-g(\lambda(x))-c^{\prime}(x) \lambda(x)+c\left(x^{*}\right) .
$$

In particular, (23) is always true when $x=x^{*}$.

Proof. we consider three cases.

Case 1. $q^{*}>\lambda(x)$. In this case $\pi^{*}(x) \leq v^{*}(x, \omega)$. Then $l^{*}(x, \omega)=0$. Notice that this case would not happen when $x=x^{*}$.

Case 2. $q^{*}=\lambda(x)$. In this case,

$$
l^{*}(x, \omega)=\left\{\begin{array}{l}
\left(\rho^{*}+\eta(x)-h-p\right)^{+}, \quad \text { if } d(\omega)=\lambda(x)=q^{*} \\
0, \quad \text { otherwise, }
\end{array}\right.
$$

If $\rho^{*}+\eta(x)-h-p \leq 0$, then $l^{*}(x, \omega)=0$. Therefore, we assume $\rho^{*}+\eta(x)-h-p>0$ which implies that $\eta(x)>0$. Then, because $q^{*}=\lambda(x)$, we have

$$
\begin{aligned}
\mathrm{E}\left[l^{*}(x, \omega) d(\omega)\right] & =q^{*}\left(F\left(q^{*}\right)-F_{-}\left(q^{*}\right)\right) \cdot\left(\rho^{*}+\eta(x)-h-p\right) \\
& =q^{*}\left(F\left(q^{*}\right)-F_{-}\left(q^{*}\right)\right) \rho^{*}+\lambda(x)\left(F(\lambda(x))-F_{-}(\lambda(x))\right) \cdot(\eta(x)-h-p) .
\end{aligned}
$$

By $(22)$ and the assumption $q^{*}=\lambda(x)$, it follows that

$$
q^{*}\left(F\left(q^{*}\right)-F_{-}\left(q^{*}\right)\right) \rho^{*}=c\left(x^{*}\right)-(p+h) \mathrm{E}\left[d(\omega) \delta\left(d(\omega) \in\left(\lambda(x), x^{*}\right]\right)\right]-x^{*}\left(p-(p+h) F\left(x^{*}\right)\right) .
$$

On the other hand, by the definition of $\eta(x)$ and the fact that $\eta(x)>0$, we have

$$
\lambda(x)\left(F(\lambda(x))-F_{-}(\lambda(x))\right) \cdot(\eta(x)-h-p)=\lambda(x)\left(p-c^{\prime}(x)-(p+h) F(\lambda(x))\right) .
$$

Combining (24) and (25), we get

$$
\begin{aligned}
& \mathrm{E}\left[l^{*}(x, \omega) d(\omega)\right] \\
= & c\left(x^{*}\right)-(p+h) \mathrm{E}\left[d(\omega) \delta\left(d(\omega) \in\left(\lambda(x), x^{*}\right]\right)\right]-x^{*}\left(p-(p+h) F\left(x^{*}\right)\right) \\
& +\lambda(x)\left(p-c^{\prime}(x)-(p+h) F(\lambda(x))\right) \\
= & c\left(x^{*}\right)-c^{\prime}(x) \lambda(x) \\
& -(p+h) \mathrm{E}\left[d(\omega) \delta\left(d(\omega) \in\left[0, x^{*}\right]\right)\right]-x^{*}\left(p-(p+h) F\left(x^{*}\right)\right) \\
& +(p+h) \mathrm{E}[d(\omega) \delta(d(\omega) \in[0, \lambda(x)])]+\lambda(x)(p-(p+h) F(\lambda(x))),
\end{aligned}
$$


from which, and by using Lemma 3, (23) follows.

To see that (23) holds when $x=x^{*}=\lambda\left(x^{*}\right)=q^{*}$, it is sufficient to consider the case when $\rho^{*}+\eta(x)-h-p \leq 0$ and thus $l^{*}(x, \omega)=0$. In this case, the left hand side of (23) is equal to 0 . On the other hand, by Lemma $5, \rho^{*}+\eta(x)-h-p \leq 0$ implies $\left(F\left(x^{*}\right)-F_{-}\left(x^{*}\right)\right) x^{*}=0$. When $x^{*}=0, c\left(x^{*}\right)-c^{\prime}\left(x^{*}\right) x^{*}=0$ holds automatically. Otherwise, $F\left(x^{*}\right)=F_{-}\left(x^{*}\right)$ and thus (20) holds, which further implies that

$$
x^{*}\left(p-c^{\prime}\left(x^{*}\right)-(p+h) F\left(x^{*}\right)\right)=c\left(x^{*}\right)-c^{\prime}\left(x^{*}\right) x^{*},
$$

and thus $c\left(x^{*}\right)-c^{\prime}\left(x^{*}\right) x^{*}=0$.

Case 3. $q^{*}<\lambda(x)$. In this case,

$$
l^{*}(x, \omega)= \begin{cases}0 & \text { if } d(\omega)<q^{*} \text { or } d(\omega)>\lambda(x) ; \\ \rho^{*} & \text { if } d(\omega)=q^{*} \\ (p+h) & \text { if } q^{*}<d(\omega)<\lambda(x) \\ \eta(x) & \text { if } d(\omega)=\lambda(x) .\end{cases}
$$

Moreover, because $\lambda(x)>q^{*} \geq 0$, by Lemma 1 and the definition of $\eta(x)$, we have,

$$
\eta(x) \lambda(x)\left(F(\lambda(x))-F_{-}(\lambda(x))\right)=\lambda(x)\left(p-c^{\prime}(x)-(p+h) F_{-}(\lambda(x))\right) .
$$

Thus

$$
\begin{aligned}
& \mathrm{E}\left[l^{*}(x, \omega) d(\omega)\right] \\
= & q^{*}\left(F\left(q^{*}\right)-F_{-}\left(q^{*}\right)\right) \rho^{*}+(p+h) \mathrm{E}\left[d(\omega) \delta\left(d(\omega) \in\left(q^{*}, \lambda(x)\right)\right)\right]+\eta(x) \lambda(x)\left(F(\lambda(x))-F_{-}(\lambda(x))\right) \\
= & c\left(x^{*}\right)-(p+h) \mathrm{E}\left[d(\omega) \delta\left(d(\omega) \in\left(q^{*}, x^{*}\right]\right)\right]-x^{*}\left(p-(p+h) F\left(x^{*}\right)\right) \\
& +(p+h) \mathrm{E}\left[d(\omega) \delta\left(d(\omega) \in\left(q^{*}, \lambda(x)\right)\right)\right]+\lambda(x)\left(p-c^{\prime}(x)-(p+h) F_{-}(\lambda(x))\right) \\
= & c\left(x^{*}\right)-c^{\prime}\left(x^{*}\right) x^{*} \\
& -(p+h) \mathrm{E}\left[d(\omega) \delta\left(d(\omega) \in\left[0, x^{*}\right]\right)\right]-x^{*}\left(p-c^{\prime}\left(x^{*}\right)-(p+h) F\left(x^{*}\right)\right) \\
& +(p+h) \mathrm{E}[d(\omega) \delta(d(\omega) \in[0, \lambda(x)])]+\lambda(x)\left(p-c^{\prime}(x)-(p+h) F(\lambda(x))\right),
\end{aligned}
$$

Similar to Case 2, by using Lemma 3, (23) follows.

Now we are ready to prove that $\left(\pi^{*}, v^{*}, l^{*}\right)$ is an optimal solution to (Concave-D). 
We first prove that $\left(\pi^{*}, v^{*}, l^{*}\right)$ is feasible to (Concave-D). By definition, $\left(\pi^{*}, v^{*}, l^{*}\right)$ satisfies constraint (14). Constraints (16) and (17) are satisfied because from Theorem $3, v^{*}(x, \omega)$ is an optimal solution thus feasible solution to $\left(\mathbf{C D L}_{x}\right)$ for any $x$.

To verify constraint (15), we notice that if $l^{*}(x, \omega)=0$, then

$$
\mathrm{E}\left[l^{*}(x, \omega) d(\omega)\right]=0 \leq c(x)-c^{\prime}(x) x
$$

Otherwise, by Lemma 6 ,

$$
\mathrm{E}\left[l^{*}(x, \omega) d(\omega)\right]=g\left(x^{*}\right)-g(\lambda(x))-c^{\prime}(x) \lambda(x)+c\left(x^{*}\right) .
$$

But, by the definition of $x^{*}$,

$$
g\left(x^{*}\right)+c\left(x^{*}\right) \leq g(\lambda(x))+c(\lambda(x)) .
$$

Then we have

$$
\mathrm{E}\left[l^{*}(x, \omega) d(\omega)\right] \leq c(\lambda(x))-c^{\prime}(x) \lambda(x)
$$

By concavity of function $c(x)$, we have

$$
c(\lambda(x)) \leq c(x)+c^{\prime}(x)(\lambda(x)-x)
$$

which, together with (26), implies that constraint (15) is satisfied for any $x \geq 0$. This completes the proof that $\left(\pi^{*}, v^{*}, l^{*}\right)$ is feasible to (Concave-D).

Now we consider the objective value corresponding to $\left(\pi^{*}, v^{*}, l^{*}\right)$. First notice that, if $q^{*}<$ $\lambda\left(x^{*}\right)=x^{*}$, then

$$
\pi^{*}(\omega) \geq v^{*}\left(x^{*}, \omega\right) .
$$

If $q^{*}=x^{*}$ and $\left(F\left(x^{*}\right)-F_{-}\left(x^{*}\right)\right) x^{*}=0$, then

$$
\pi^{*}(\omega) d(\omega) \geq v^{*}\left(x^{*}, \omega\right) d(\omega)
$$

On the other hand, if $q^{*}=x^{*}$ and $\left(F\left(x^{*}\right)-F_{-}\left(x^{*}\right)\right) x^{*}>0$, then by Lemma $5, \rho^{*}+\eta\left(x^{*}\right) \geq p+h$. Thus, in this case, $\pi^{*}(\omega) \geq v^{*}\left(x^{*}, \omega\right)$. Therefore, no matter what cases, we have that, $\pi^{*}(\omega) d(\omega) \geq$ $v^{*}\left(x^{*}, \omega\right) d(\omega)$ almost surely, which implies that

$$
\pi^{*}(\omega) d(\omega)=v^{*}\left(x^{*}, \omega\right) d(\omega)+l^{*}\left(x^{*}, \omega\right) d(\omega),
$$


and thus

$$
\mathrm{E}\left[\pi^{*}(\omega) d(\omega)\right]=\mathrm{E}\left[v^{*}\left(x^{*}, \omega\right) d(\omega)\right]+\mathrm{E}\left[l^{*}\left(x^{*}, \omega\right) d(\omega)\right] .
$$

From Lemma 6 and the fact that $x^{*}=\lambda\left(x^{*}\right)$, we get

$$
\mathrm{E}\left[l^{*}\left(x^{*}, \omega\right) d(\omega)\right]=-c^{\prime}\left(x^{*}\right) \lambda\left(x^{*}\right)+c\left(x^{*}\right) .
$$

On the other hand, from Theorem 3 , and by the definition of $v^{*}\left(x^{*}, \omega\right)$, we know that $v^{*}\left(x^{*}, \omega\right)$ is an optimal solution to $\left(\mathbf{C D L}_{x^{*}}\right)$. Then it follows from the strong duality between $\left(\mathbf{C D L}_{x^{*}}\right)$ and $\left(\mathbf{C P L}_{x^{*}}\right)$, that

$$
\mathrm{E}\left[v^{*}\left(x^{*}, \omega\right) d(\omega)\right]=c^{\prime}\left(x^{*}\right) x^{*}+g\left(x^{*}\right) .
$$

Therefore, we have

$$
\mathrm{E}\left[\pi^{*}(\omega) d(\omega)\right]=c\left(x^{*}\right)+g\left(x^{*}\right)
$$

where the right hand side is the optimal value of (Concave-P). The proof is complete. 